\section{Cahiers de Narratologie}

Analyse et théorie narratives

39 | 2021

L'héritage de Ricoeur : du récit à l'expérience

\title{
Raphaël Baroni et Claus Gunti (dir), Introduction aux cultures numériques,
}

\section{Karl Akiki}

\section{(2) OpenEdition}

\section{Journals}

Édition électronique

URL : https://journals.openedition.org/narratologie/12155

DOI : 10.4000/narratologie. 12155

ISSN : 1765-307X

Éditeur

LIRCES

\section{Référence électronique}

Karl Akiki, «Raphaël Baroni et Claus Gunti (dir), Introduction aux cultures numériques, », Cahiers de Narratologie [En ligne], 39 | 2021, mis en ligne le 11 juin 2021, consulté le 21 juillet 2021. URL : http:// journals.openedition.org/narratologie/12155; DOI : https://doi.org/10.4000/narratologie.12155

Ce document a été généré automatiquement le 21 juillet 2021.

Article L.111-1 du Code de la propriété intellectuelle. 


\title{
Raphaël Baroni et Claus Gunti (dir), Introduction aux cultures numériques,
}

\author{
Karl Akiki
}

\section{RÉFÉRENCE}

Armand Colin, 2020, 394p., ISBN : 978-2-200-62885-7

\section{Aperçu général}

1 Le domaine des humanités numériques met, aujourd'hui, dos à dos deux catégories de chercheurs : ceux qui s'ancrent dans la tradition des sciences humaines pour en faire un pilier de réflexion permettant d'aborder la transition numérique de la culture; et ceux qui embrassent les nouvelles technologies en les implémentant dans leurs disciplines respectives (accès aux sources, analyse quantitative des objets, visualisation des données...). Le hiatus entre ces deux manières d'appréhender les questions relatives à la numérisation de la culture est d'une grande importance épistémologique pour les humanités.

Introduction aux cultures numériques, dirigé par Raphaël Baroni et Claus Gunti, naît de ce clivage. Autour de cette problématique qui naît de l'intrusion du numérique dans la culture, se réunissent des chercheuses et des chercheurs venant d'horizons divers (sociologie des médias, études culturelles, narratologie, études théâtrales...). Un mouvement double sous-tend l'armature de la réflexion critique: comment les technologies numériques ont-elles bouleversé l'horizon culturel? comment la culture contemporaine est-elle remodelée par le numérique?

3 L'ouvrage répond à cette interrogation en trois parties et en 17 chapitres pris en charge par des auteurs différents. Dans une première partie ( La Transition numérique des médias »), sept médias (littérature, théâtre, bande dessinée, photographie, cinéma, télévision et jeux vidéo) sont étudiés dans leur rapport à l'irruption des technologies 
numériques. Cette mise en perspective permet de comprendre comment chaque média a réagi à la transition numérique. Certains ont intégré cette mutation avec aisance, d'autres y ont opposé une résistance particulière, notamment ceux liés la culture du livre. Cette partie s'intéresse également à certains marqueurs médiatiques qui ne peuvent pas être numérisables (la performance théâtrale, la salle obscure, la fixité de la page imprimée...). Les contributions posent donc un regard nuancé sur les conséquences de la numérisation tant au niveau de la production, de la distribution que de la consommation des objets numériques. Cela permet ainsi de mieux comprendre le pluriel apposé dans le titre au terme « culture ».

4 La deuxième partie ("Des Médias au transmédia») réfléchit à la convergence médiatique et aux différentes déclinaisons de la notion de narration transmédiale ainsi qu'à à ses effets sur la culture. Le propos s'organise en quatre chapitres constitués notamment, d'une traduction, inédite en français, de trois textes extraits du blog de Henry Jenkins. L'AcaFan s'y intéresse à la logique intrinsèque la création des dispositifs transmédiatiques et à leurs usages par les fans. Le quatrième chapitre, pris en charge par Baroni, s'interroge sur la constitution et l'organisation des « constellations ${ }^{1}$ » de récits produits à l'ère du transmédia. Un double mouvement anime cette réflexion qui prend appui sur la construction des rapports entre le centre d'un univers narratif et sa périphérie, avant d'effectuer une évaluation concernant l'importance de l'intrigue dans l'évaluation des hiérarchies narratives.

5 La troisième partie (" Nouveaux médias, nouveaux paradigmes ») met en lumière une série de nouvelles problématiques liées à l'émergence du numérique. Les six chapitres, sans lien véritablement apparent, reviennent sur la transfictionnalité, phénomène accéléré par la mise en réseau de la culture et qui survient lorsque « deux textes, du même auteur ou non, se rapportent à une même fiction » (R. Saint Gelais, chapitre 12, p.252) ; sur la remédiatisation, c'est-à-dire sur le processus d'apparition de nouveaux médias qui permettent une redéfinition des œuvres et des anciens médias, partiellement intégrés dans cette mutation médiatique (J. Baetens, chapitre 13) ; sur la quête technologique d'une immersion idéale (M.-L. Ryan, chapitre 14); sur les effets spéciaux liés aux trucages numériques dans le cinéma spectaculaire (A. Boillat, chapitre 15); sur l'émergence d'une critique plurielle en ligne qui court-circuite les acteurs traditionnels singuliers (S. Estier, chapitre 16); et sur la place que l'enseignement de la littérature pourrait occuper dans l'univers des techno-images (Y. Citton, chapitre 17).

6 Cet ouvrage, pionnier dans sa manière polyphonique de prendre la transition numérique à bras-le-corps, met en exergue les changements culturels liés à l'émergence du numérique et à l'expansion galopante de ces technologies. Ces bouleversements s'affirment particulièrement au niveau des démarcations qui organisent le paysage culturel, de la modification des frontières ${ }^{2}$ technologiques qui remodèle les rapports entre ceux qui produisent l'objet culturel et ceux qui le consomment.

\section{Bouleversements des frontières technologiques}

Comment le numérique a-t-il transformé le rapport de la culture à la technologie? Les trois parties d'Introduction aux cultures numériques tentent de répondre à cette question en analysant en profondeur des éléments précis, voire insoupçonnés, qui sont la marque de fabrique de cette évolution. On peut dès lors étendre le propos captivant de 
J. Baudry concernant la bande dessinée (chapitre 3) à l'ensemble de la culture en notant que le numérique exerce une double influence.

8 Cela commence dans un premier temps au niveau du processus de création. Les créateurs des médias contemporains (auteurs, metteurs en scènes, cinéastes, bédéistes...) disposent de nouveaux outils de production et de moyens multiples pour interagir avec leurs publics. En littérature (B. Gervais et S. Brousseau, chapitre 1), on constate ainsi la multiplication des blogs d'écrivains, la présence massive des auteurs sur les réseaux sociaux, la programmation de contenus inédits renforçant des pratiques de lecture " créative » (Citton, chapitre 17). Au théâtre (D. Chaperon et I. Pluta, chapitre 2), média que l'on pourrait croire - a priori - hermétique au numérique -, c'est, d'un côté, la "digital performance " (Dixon, 2007) qui marque l'apparition des écrans, des visiocasques et autres drones dans le paysage esthétique; et de l'autre, de manière moins apparente, c'est le travail en régie qui devient en lui-même une création entièrement numérisée (ce qui réduit les aléas dus aux facteurs humains nonmaitrisables). La bande dessinée (J. Baudry, chapitre 3) a, quant à elle, incorporé le geste numérique depuis les années 80 grâce aux logiciels de dessin assisté (lettrage, colorisation) et aux interfaces graphiques. Pour la photographie (C. Gunti, chapitre 4), le cinéma (Ph. Marion, chapitre 5; A. Boillat, chapitre 15) ou la télévision (F. Jost, chapitre 6), le numérique permet le trucage qu'il ontologise, élargissant les horizons du processus créatif. Dans un siècle hyper visualisé et vocal, les technologies numériques permettent ainsi à la création artistique d'interroger les concepts de mimésis, d'interactivité et de prise en compte du pôle esthétique quand on envisage les potentialités du support matériel de l'œuvre culturelle.

9 Cette réflexion aboutit, dans un second temps, à aborder le processus de diffusion. La remédiation ( façon dont un médium est perçu par notre culture comme une transformation ou une amélioration par rapport à un autre », Bolter et Grusin 2000 in J. Baetens, chapitre 13) met en lumière la remodélisation - comment l'apparition d'un média renouvelle un autre média en l'englobant partiellement. Les billetteries en ligne, les trailers pour des pièces de théâtre, l'utilisation massive des Black mirrors (écrans de tablettes ou de smartphones) comme substitut à l'écran de télévision) sont autant de moyens de facilitation et de diversification des actes de consommation culturelle. Ces nouvelles technologies constituent ainsi, une invitation à la démocratisation de la culture laquelle est devenue accessible en un clic, à tout moment, en tout lieu et par tout un chacun... en un mot, le syndrome ATAWAD dans toute sa splendeur (anytime, anywhere, any device).

10 Grâce aux nouvelles technologies, le numérique étend l'éventail des choix médiatiques et constitue un réseau de plus en plus serré d'expériences diversifiées et coordonnées. L'expérience culturelle du récepteur (lecteur, spectateur, téléspectateur...) est ainsi modifiée pour peu qu'il sache trouver sa voie entre "technophobie» et « technophilie » (Simondon, 1989), pour peu qu'il accepte le concept de responsabilité numérique de ses choix culturels offerts en open access, éloignés des diktats des bienpensants - ces enjeux éthiques sont d'ailleurs abordés aux deux derniers chapitres (S. Estier, chapitre 16 et $Y$. Citton, chapitre 17). 


\section{Bouleversements des frontières narratives}

11 Pour se rassurer, d'aucuns auraient tendance à limiter l'impact des mutations technologiques liées au numériques à l'univers médiatique, à des mutations d'ordre formel ou économique. Introduction aux cultures numériques va plus loin (surtout dans sa deuxième partie) et ose le pari d'approfondir la réflexion pour établir clairement les incidences du numérique sur la prise en charge de l'intrigue, sur le récit et sur la diversification des supports de la narration.

12 Il faut d'abord rappeler que les horizons qu'ouvre ce nouveau virage existaient déjà dans le domaine de la littérature analogique. Le concept d'hyperliens qui tend à se généraliser, n'est-il pas l'incarnation de la théorie de Bremond concernant les possibles narratifs (B. Gervais et $\mathrm{S}$. Brousseau, chapitre 1) ? Le théâtre classique et ses règles ne sont-ils pas la figuration de la mathématisation rendue possible par le numérique (D. Chaperon et I. Pluta, chapitre 2)?

13 À l'ère du numérique, les nouveaux récits prennent souvent appui sur la transmédialité particulièrement lorsqu'ils s'inscrivent dans une logique sérielle. Celle-ci a l'avantage de faire éclater l'acte de lecture (H. Jenkins, chapitre 9) étant donné que, pour que le lecteur puisse maîtriser tous les tenants et aboutissants de l'univers fictionnel, il devrait idéalement se référer à une multitude de supports éparpillés. L'imaginaire de la fiction s'en trouve ainsi épaissi puisque ce sont les mondes créés par les différents médias qui sont alors mis en avant (A. Boillat, chapitre 15). Les limites entre texte, péritexte et paratexte telles que proposées par Genette (R. Baroni, chapitre 11) sont remises en question et de nombreux éléments peuvent entrer au service de la construction d'univers fictionnels aux embranchements multiples.

14 Cette narrativité 2.0 est à étudier dans ses incidences sur l'acte de lecture. Le public voit augmenter sa marge de manœuvre quand il s'agit de parcourir les univers narratifs, ou de ralentir, de relancer, d'accélérer le visionnage d'un contenu audio-visuel (F. Jost, chapitre 6). Il se soumet à une nouvelle " expérience narrative » (M.-L. Ryan, chapitre 14) qui suppose une culture encyclopédique (H. Jenkins, chapitre 9) et une ouverture au jeu favorisée par l'immersion (Krichane, chapitre 16). C'est dans cette optique que le numérique passe au service du narratif.

\section{Bouleversements des frontières de la réception}

15 Tous les auteurs de l'ouvrage l'affirment sans équivoque : le numérique permet à la culture de ne plus être une nébuleuse insaisissable et imposée. Le récepteur y est pris en compte : il a son mot à dire.

Comme l'affirme $\mathrm{H}$. Jenkins au chapitre 8 , les médias populaires ont pu souffrir, jusqu'à une période récente, du regard condescendant que porte sur eux le monde académique. Avant l'émergence de ces travaux, les fans étaient perçus comme de simples consommateurs décérébrés, des récepteurs passifs se contentant d'ingurgiter la culture par télécommande ou téléphone interposés. Cette image est pourtant une caricature hâtive de la réalité. Avec les cultures numériques, le lecteur se rebelle : il a enfin un rôle à jouer, il délaisse sa passivité traditionnelle et veut faire entendre sa voix.

17 Cela se manifeste notamment à travers la narration transmédiatique qui fait émerger une nouvelle catégorie, celle des « chasseurs-cueilleurs" (H. Jenkins, chapitre 10) qui 
vont à la recherche des éléments de l'intrigue en naviguant à travers différents médias pour reconstituer la cohérence de la cartographie d'un univers narratif. Plus encore, cette activité ne s'effectue plus d'une manière solitaire mais en interaction avec l'ensemble de la communauté de fans qui abandonnent l'anonymat et s'affirment au grand jour (Saint-Gelais, chapitre 12). Ils réclament leur part de l'acte créatif (qui s'exprime notamment via la fanfiction), ils imposent une culture participative qui rend plus poreuses les frontières entre le réel et le virtuel. Leurs demandes sont dès lors prises en compte par les artistes, les producteurs et les diffuseurs qui multiplient les moyens leur permettant de s'exprimer. On constate ce phénomène à travers l'importance grandissante que prend aujourd'hui la critique en ligne (Estier, chapitre 16) : avis et commentaires des consommateurs font partie intégrante du paysage culturel contemporain. Les univers fictionnels, quant à eux, expérimentent un processus d'expansion continue que nourrissent les apports d'acteurs différenciés. L'effet «boule de neige » - selon la formule de Marie-Laure Ryan (R. Baroni, chapitre 11) - est activé grâce à un agglomérat aux contours incertains auxquels participent les fans en ajoutant leurs extensions non canoniques. C'est en rapport avec ce dernier élément que se développent aujourd'hui le primat de l'interactivité et celui de l'immersion. Ils permettent aux trois pôles de la triade culturelle (le créateur, le diffuseur et le récepteur) de resserrer leurs liens et de redéfinir leurs rôles respectifs.

On le voit, Introduction aux cultures numériques devrait constituer un jalon important dans le monde de la recherche académique francophone. Les contributions de ces chercheuses et chercheurs prouvent qu'il faut, aujourd'hui, prendre en considération les évolutions de la sphère culturelle. Elles ouvrent la voie à une inflexion nécessaire des objets traditionnels de la recherche académique aux enjeux qui intéressent la cité.

\section{BIBLIOGRAPHIE}

BESSON, A. (2015), Constellations. Des mondes fictionnels dans l'imaginaire contemporain, Paris, CNRS Éditions.

BOURDIEU, P. (1979), La Distinction. Critique sociale du jugement, Paris, Éditions de Minuit.

BREMOND, C. (1971), « Observations sur la Grammaire du Décaméron », Poétique, n6.

SIMONDON, G (1989), L'Individuation psychique et collective, Paris, Aubier.

\section{NOTES}

1. Baroni reprend le terme d'Anne Besson (Constellations. Des mondes fictionnels dans

l'imaginaire contemporain, 2015).

2. Ce terme est à comprendre dans son sens bourdieusien (Bourdieu, 1979). 


\section{AUTEUR}

KARL AKIKI

Université Saint-Joseph de Beyrouth (Liban) 